\title{
Surface enhanced imaging and IR spectroscopy of the biological cells on the nanostructured gold film
}

\author{
G.I. Dovbeshko' ${ }^{1}$, O.P. Gnatyuk ${ }^{1}$, S.O. Karakhim² ${ }^{2}$ T.P. Doroshenko ${ }^{3}$, V.R. Romanyuk ${ }^{3}$ \\ ${ }^{I}$ Institute of Physics, NAS of Ukraine, \\ 46, prospect Nauky, 03680 Kyiv, Ukraine, \\ Phone: +38 (044) 525-98-51, fax: +38 (044)525-15-89, \\ e-mail: gd@iop.kiev.ua \\ ${ }^{2}$ Palladin Institute of Biochemistry, NAS of Ukraine, \\ 9, Leontovycha str., 01601 Kyiv, Ukraine \\ ${ }^{3} V$. Lashkaryov Institute of Semiconductor Physics, NAS of Ukraine, \\ 41, prospect Nauky, 03680 Kyiv, Ukraine
}

\begin{abstract}
New approach for optical imaging, structural study and cell cultivation based on the effect of the enhancement of optical signals from biomolecules and biological cells near nanostructured rough gold surface is proposed. The surface enhanced IR absorption (SEIRA) spectroscopy and confocal microscopy experiments were made using the culture of SPEV (porcine embryonic kidney epithelium transplantable line) and fibroblast cells, cultivated and/or adsorbed on the gold substrate. The SEIRA spectra registered from monolayer of the SPEV cells cultivated on the rough gold showed a low frequency shift of about 2 to $7 \mathrm{~cm}^{-1}$ for the most characteristic IR vibrations, compared with those adsorbed from suspension on the same substrate. An enhancement factor of $15 \ldots 30$ was obtained for different molecular vibrations. The confocal microscopy contrast images of the SPEV cells on rough gold substrate were obtained in laser fluorescence mode. This approach opens new possibilities for visualization of the living cells in vivo without staining. The fluorescence of the rough gold surfaces and effects responsible for our findings have been discussed.
\end{abstract}

Keywords: SPEV cells, nanostructured rough gold substrate, SEIRA spectroscopy, laser scanning confocal microscopy, fluorescence imaging.

Manuscript received 16.11.16; revised version received 19.04.17; accepted for publication 14.06.17; published online 18.07.17.

\section{Introduction}

Contrast imaging of biological molecules and cells is of great importance for medical and biological analysis. However, optical transparency and weak fluorescence of biological objects restricts microscopic imaging. Therefore, various markers such as dyes, quantum dots, nanoparticles are used for improvement of cell visualization. For example, a dye molecules of green fluorescent protein (GNP) are used to mark a cytoskeleton, molecules of DAPI (4",6-diamidino-2phenylindole) - to stain a nuclei in the cell. At the same time, development of the free-label method is attractive for non-destructive cell analysis and has grown recently.

IR spectroscopy methods, with their increased sensitivity (i.e., signal-to-noise ratio), are essential for structural studies of biological objects. It is known that optical signals from the molecules adsorbed on the nanostructured metal substrate or colloidal metal nanoparticles can be enhanced by 10 to $10^{3}$ times for infra-red (IR) absorption [1, 2], by $\sim 10^{3}$ for luminescence, and by $\sim 10^{6}$ for Raman scattering (RS). Earlier, we have reported an enhancement of IR signals for Amid I band from albumin for a near monomolecular layer by more than 100 times [3] as well as essential enhancement in our SEIRA study of phospholipids [4]. These data are in accordance with other publications on SEIRA effect [5-8]. We have got an enhancement factor of about $10^{5}$ in SERS spectra of poly-A molecules adsorbed on carbon nanotubes and deposited on commercially available SERS substrate [9]. So, if it is possible to obtain the signal from different cellular 
components enhanced by rough gold surface, we would expect to obtain an enhanced IR absorption signal, i.e. SEIRA spectra, from the whole cells deposited on the rough metal surface.

The second point is related with a new approach to optical microscopy visualization of the cells deposited on the same gold substrates, which was applied for SEIRA spectroscopy. Laser scanning confocal microscopy (LSCM) was chosen as one of the modern and widely-used instrument for imaging of biological structures and processes in different branches of medicine, biology and bionanotechnology. Confocal imaging is related to fluorescent methods. The objects under study should possess proper luminescence or should be stained with fluorescent probes and be transparent for light penetration to a certain depth. Fluorescent confocal microscopy was proved to be a powerful tool to study alive and fixed cells, thin cuts of plant and animal tissues and even small organisms. It is especially important for studying the alive cells to have a possibility to provide imaging with high spatial and time resolutions. It enables to clear up the most complicated organization of the whole organism at molecular, cellular and tissue levels.

However, an application of fluorescent probes for studying the cell structure has a number of disadvantages. First, this is cytotoxicity in the case of alive cells, non-selectivity of the probes to sub-cell components, as well as insufficient specificity and overlapping of fluorescence spectra from different probes. Therefore, an actual task is a search for imaging possibilities for cells with confocal microscopy methods without application of fluorescent probes.

Here, we discuss a strategy complying SEIRA spectroscopy together with confocal microscopy to study living cells and analyze monostructured gold film for visualization of free labeling cells. The proposed new approach for structural study and visualization of cells is based on the effect of the enhancement of optical signals from molecules and cells deposited on a nanostructured gold substrate.

\section{Materials and methods}

\subsection{Sample preparations}

SPEV (porcine embryonic kidney epithelium transplantable line) cells for our experiments were cultured in plastic flasks in DMEM (Dulbecco's Modified Eagle's medium) (Sigma) medium with 5\% fetal calf serum FCS (v/v) (HyClone), kanamycin $(100 \mu \mathrm{g} / \mathrm{ml})$, amphotericin B $(5 \mu \mathrm{g} / \mathrm{ml})$ according to [10]. A total number of the grown cells was calculated using the conventional method in Goryaev chamber [11]. Sown concentration was equal to $5 \times 10^{4}$ cells $/ \mathrm{cm}^{2}$. The cells were grown in Sanyo incubator at the temperature $+37^{\circ} \mathrm{C}$ in humid atmosphere with $5 \% \mathrm{CO}_{2}$. Grown solution was replaced every 2 days. Cells were passaged at $\sim 80 \%$ confluence. The cultivated SPEV cells were transferred into suspension and placed on a cover glass or Au-precoated glass substrate. The samples were returned to the incubator for $24 \ldots 48 \mathrm{~h}$ to ensure adequate adhesion. Prior to physical experiments, the culture medium was removed. For confocal microscopy and FTIR experiments, the SPEV cells were cultured on a gold substrate placed in sterile Petri dishes in incubator for $24 \ldots 48$ hours. All the cell samples were washed triple in Hanks solution placed in a waterproof glass camera and dried for 1 hour at 0.5 atm under evacuation by using the Millipore XF 5423050 vacuum pump.

As a substrate, we used a gold (Au) thin $(20 \ldots 35 \mathrm{~nm})$ films fabricated by vacuum thermal deposition of pure (0.99999) Au onto glass substrates (TF-1 glass, $20 \times 20 \mathrm{~mm}$ ) preliminary covered with the intermediate $5 \mathrm{~nm}$ adhesive $\mathrm{Cr}$ layer [8]. Before $\mathrm{Au}$ deposition, glass surface was cleaned with $\mathrm{NH}_{4} \mathrm{OH}: \mathrm{H}_{2} \mathrm{O}_{2}: \mathrm{H}_{2} \mathrm{O}$ and $\mathrm{HCl}: \mathrm{H}_{2} \mathrm{O}_{2}: \mathrm{H}_{2} \mathrm{O}(1: 2: 2$ by $\mathrm{v} / \mathrm{v})$ solutions, subsequently, for $5 \mathrm{~min}$ at the boiling temperature. Then it was rinsed in bidistilled water and dried in a flow of pure nitrogen.

The morphology of the gold surface has been studied with a Nanoscope IIIa (Digital Instrument) atomic force microscope (AFM) in a tapping mode. Tapping force mode scans were performed using commercially available AFM silicon nitride tips. The scanning frequency was approximately $1 \mathrm{~Hz}$ in all experiments. Statistical analysis was made with conventional statistics program.

Reflectance and transmittance spectra of initial gold thin films were measured in the spectral range $\lambda=$ $380 \ldots 1100 \mathrm{~nm}$ with DMR-4 monochromator and variable-angle of incidence reflectance attachment in $p$ polarized light. Device is equipped with silicone photodiode photodetector and incandescent bulb lamp as wide-band continuous spectra light source.

Confocal imaging was carried out with a Carl Zeiss LSM-510 META confocal laser scanning microscope with the Plan-Achromat $63 \times / 1.4$ Oil DIC oil immersion objective in Multi Track mode. The confocal microscope optical scheme is presented in Fig. 1. A halogen incandescent lamp was used for observing objects in the transmittance mode in visible light. A fluorescence images of the objects were obtained by excitation with ultraviolet mercury lamp HBO-100 or with diode laser $(\lambda=405 \mathrm{~nm}, 25 \mathrm{~mW}), \operatorname{Ar}$ or $\operatorname{ArKr}$ laser $(\lambda=488 \mathrm{~nm}$, $30 \mathrm{~mW})$ and HeNe laser $(\lambda=633 \mathrm{~nm}, 5 \mathrm{~mW})$ in backscattering geometry. The fluorescent light was filtered with a band pass BP $420-480 \mathrm{~nm}$ or long pass LP420 nm, BP 505-530 nm or LP $505 \mathrm{~nm}$ and LP $650 \mathrm{~nm}$ filters, respectively. LSM-510 META was equipped with a 32-chanels Metadetector (polychromatic detector) and registration system AxioCam. The scanning speed depends on the resolution and for LCM 510 microscope at $512 \times 512$ resolution, it makes up about $1 / 5$ seconds on a layer [12]. Magnification in the experiments was $63 \times$ and $10 \times$. The most important 
optical advantage of confocal microscopy is that the radiated photons are focused by the objective lens on a small $(\sim 50 \mu \mathrm{m})$ detector pinhole ( 2 in Fig. 1), which limits the depth of focus. Optical thickness $d$ of slices depends on the wavelength $(\lambda)$, objective lens numerical aperture (N.A.), refractive index $(n)$, pinhole diameter $(P)$ and roughly $d \sim P n \lambda /(\text { N.A. })^{2}$. In this work, the optical slice thickness was about $0.8 \mu \mathrm{m}$.

For SEIRA experiments, the samples with the cells deposited on the rough surface of $\mathrm{Au}$ were dried liophilically. All the samples were kept in closed tight boxes with $\mathrm{P}_{2} \mathrm{O}_{5}$ water adsorbent. The spectra were collected in the $400 \ldots 7000 \mathrm{~cm}^{-1}$ region with an IFS-66 Bruker instrument with resolution of $0.5 \mathrm{~cm}^{-1}$ and wavenumber definition accuracy of not less than $0.01 \mathrm{~cm}^{-1}$. Repeatability of the frequency in our SEIRA spectra was $0.5 \mathrm{~cm}^{-1}$ and that for the absorbance - about 0.0005 .

IR spectra from molecular vibrations of cell components (proteins, nucleic acids, lipids) deposited on gold rough surfaces were measured in external reflection configuration at light incidence angle $16.5^{\circ}$, as described in $[1-3]$.

Deconvolution and decomposition of the spectral bands were performed with Opus-5.5 software program. The peak positions of the absorption bands were estimated by using the second derivative method and/or the standard method. All the spectra were baselinecorrected and normalized by the peak intensity of $\mathrm{O}-\mathrm{H}$ stretching vibration band centred near $3300 \mathrm{~cm}^{-1}$. Estimation of the SEIRA enhancement factor was carried out as in [2].

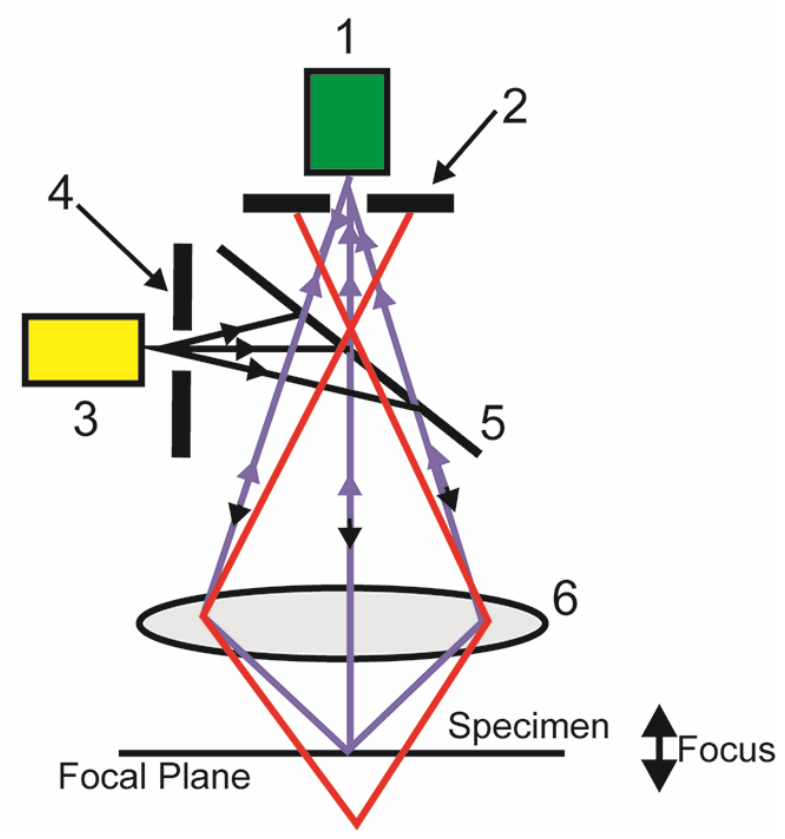

Fig. 1. Scheme of confocal microscopy: photodetector (1), detector pinhole (2), laser or light source (3), source pinhole (4), beamsplitter (5), objective lens (6).

\section{Physical basis of the effect of enhancement of optical processes}

\subsection{SEIRA}

For the first time, an enhancement of IR signal from molecules chemisorbed on $\mathrm{Au}$ and $\mathrm{Ag}$ surface by the factor up to $10^{3}$ has been registered by Harstein and colleagues in 1980 [13]. Only at the beginning of the 90th years, scientists came back to study of the enhanced IR effects, mainly due to very low limit of the molecules that could be detected by IR spectroscopy and its some advantages (non-destructivity, higher signal-noise ratio, less distorted spectra) in comparison with SERS. In 1991, the effect was named as SEIRA by Osawa and Ikeda [14]. The effect cannot be explained in a simple way, it includes several mechanisms, namely: 1) local increase of electromagnetic field near the rough metal surface or metal film due to excitation of surface plasmons, 2) increase of dipole transition moment of adsorbed molecules $[15,16]$.

The conditions for surface plasmon excitation at the interface between two media ( 1 - environment, 2 plasmon-carrying media) are as follows:

$\varepsilon_{2}^{\prime}(\lambda)<-\varepsilon_{1}^{\prime}(\lambda)$,
$\left|\varepsilon_{2}^{\prime}(\lambda) / \varepsilon_{2}^{\prime \prime}(\lambda)\right|^{2}>>1$,

where $\varepsilon^{\prime}$ and $\varepsilon^{\prime \prime}$ are real and imaginary parts of dielectric permittivity, $\lambda$ is the wavelength.

When incident light promotes excitation of molecules or cells adsorbed on a gold substrate, the probability of optical process near metal surface is increased due to enhancement of local electric field and density of photon states. Naturally, this effect depends on morphology of gold surface, sizes of roughness and film thickness [17-20].

\subsection{Metal-enhanced fluorescence}

Earlier [21, 22], it was shown that the intrinsic emission from DNA can be enhanced manifold by silver island film substrate. The time-resolved measurements have shown a decreased lifetime for the intrinsic DNA emission near the silver islands. These results of increased intensity and decreased lifetime indicate a metal-induced increase in the radiative rate decay of the DNA. As in the case of SEIRA spectroscopy, the main mechanism driving the enhanced photoluminescence (PL) of the molecule near metal surface is related with the enhanced density of photon states near metal surface and changes in probability of PL process (Fig. 2). In this figure, $E$ and $E_{m}$ - electric fields for transition from the ground state to the excited one without metal and with metal, respectively, $\Gamma$ - intrinsic radiative rate (without metal), $\Gamma_{m}$ - radiative rate with metal, $k_{n r}$ and $k_{m}$ - rate of non-radiative process without metal and with metal, respectively. 


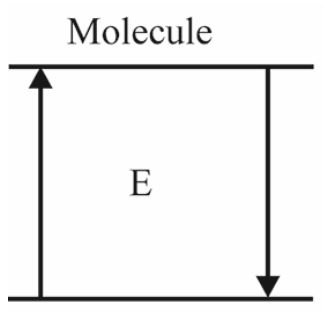

a

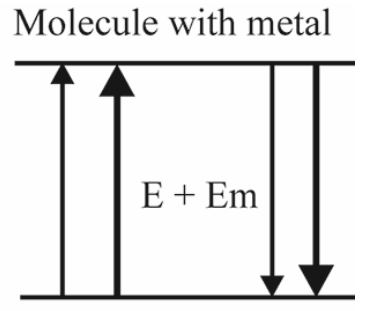

b

Fig. 2. Schematic energy diagram of fluorescence inherent to a free molecule $(a)$ and the molecule on metal surface $(b)$.

The situation with enhanced PL is more complicated in comparison with SEIRA spectroscopy due to competition between different radiative decay channels strongly influencing the PL intensity. So, in some cases for the molecules near metal surface the PL intensity would decrease [21-23]. Thus, the quantum yield, $Q_{0}$, of a fluorophore shows a competition between radiative decay and nonradiative processes $\left(k_{q}\right.$ is the rate constant of quenching):

$Q_{0}=\frac{\Gamma}{\Gamma+k_{n r}+k_{q}}$.

The fluorescence lifetime, or decay time, is the average time for an ensemble of fluorescent molecules to remain in the state:

$$
\tau_{0}=\frac{1}{\Gamma+k_{n r}+k_{q}} .
$$

When fluorophores are placed at suitable distances from metallic surface, fluorophores could undergo modifications of their radiative decay rates, where an increase in $\Gamma_{m}$ results in an increase in PL intensity, $Q_{m}$, and reduction in lifetime $\tau_{m}$, which is converse to the free-space condition. If the fluorophore quantum yield and lifetime near metal surface are expressed by the following formula

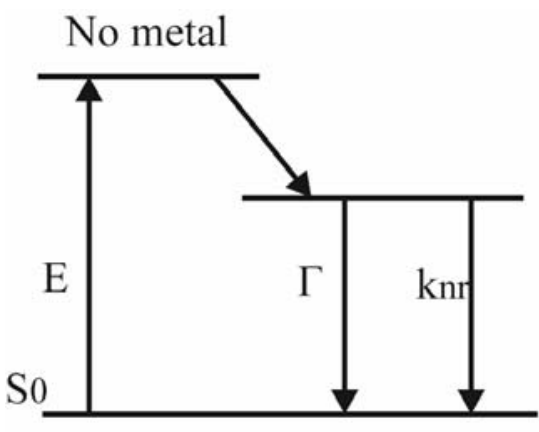

a

$$
\begin{gathered}
Q_{m}=\frac{\Gamma_{m}}{\Gamma_{m}+k_{n r}+k_{q}}, \\
\tau_{m}=\frac{1}{\Gamma_{m}+k_{n r}+k_{q}} .
\end{gathered}
$$

One can get an increase of quantum yield, when the lifetime decreases. It could be explained by strong coupling between electromagnetic field oscillation on metal surface and fluorophore dipole. In the case of metal, the increase of $Q_{m}$ caused by decrease of the lifetime $\tau_{m}$ is different in comparison with that of conventional fluorescence without fluorophore. In the latter case, $Q$ increase is due to non-radiative $k_{n}$ decay rate decrease (Fig. 3).

Therefore, near the metal surface the conditions for redistribution of channels governing by fluorescence occur. The theoretical basis and experimental evidence for PL enhancement by metal surface do exist and can manifest themselves in a number of cases.

\section{Results and discussion}

\subsection{Gold substrate for enhanced infrared spectra and luminescent imaging}

Morphology of the semitransparent gold film surface is presented in Fig. 4a. The surface relief of the $\mathrm{Au}$ substrates was found with AFM to have a rms roughness of $2 \ldots 5 \mathrm{~nm}$. Transmittance $(\mathrm{T})$, reflectance $(\mathrm{R})$ and absorption (Abs = 1-R-T) spectra (Fig. 4b) of films used indicate that films are continuous, with optical parameters close to bulk gold. The increase of absorption at $\lambda<500 \mathrm{~nm}$ is caused by intraband absorption in the gold [24]. Absorption from local plasmons (radiative modes, typically observed in the Drude region $\lambda>500 \mathrm{~nm}$ ) with isolated gold nanoparticles is not monitored. Thus, the enhancement of electromagnetic field near the metal surface can be invoked by surface roughness mainly [25].

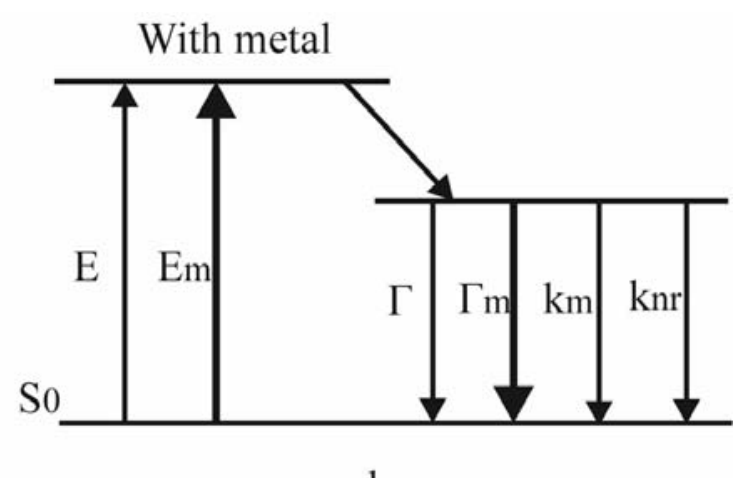

$\mathrm{b}$

Fig. 3. Classical Jablonski diagram for PL of the molecule in free space $(a)$ and its modified form in the presence of metallic surface $(b)$ : $E$ - incident photon, $E_{m}$ - concentrated by metal incident field, $\Gamma$ - radiative or emission decay rate, $\Gamma_{m}-$ radiative decay rate in the presence of metal, $k_{n r}$ and $k_{m}$ - nonradiative decay rate represents all the other rate processes returning the molecule to the ground state without emission, without and with metal, accordingly. 

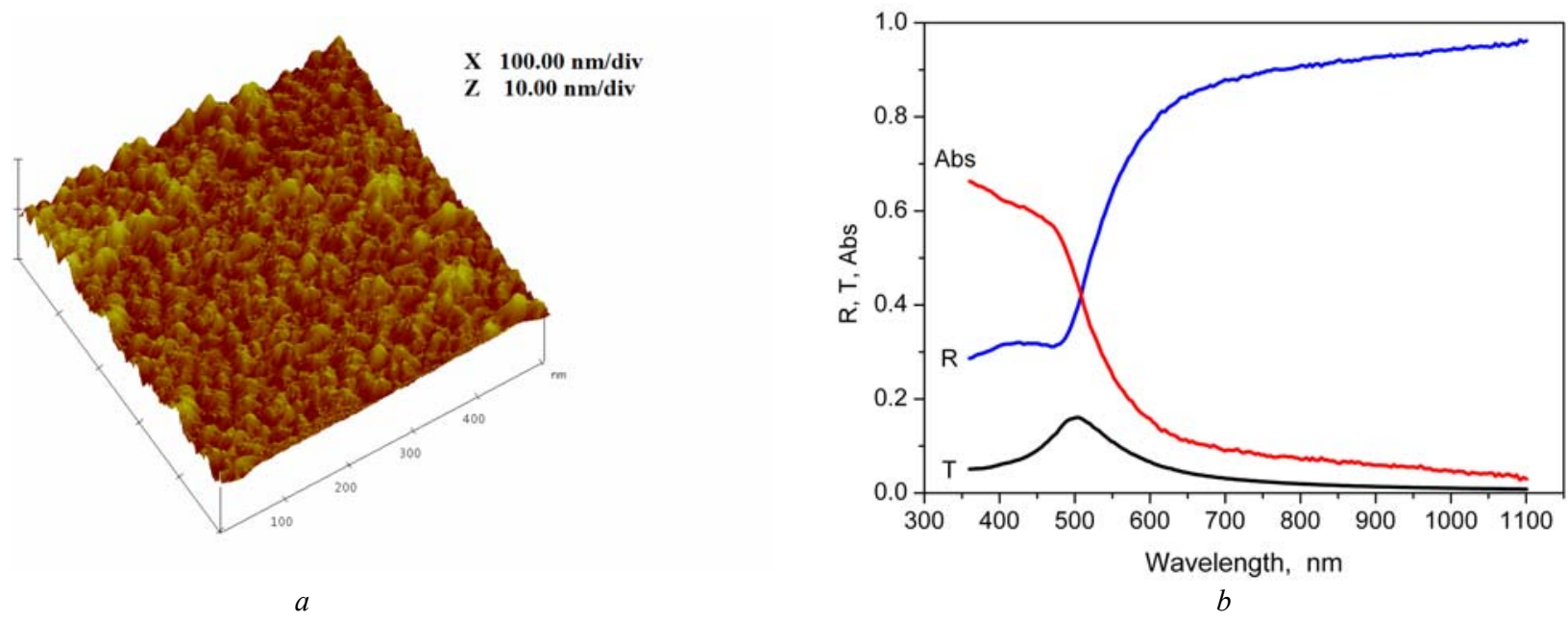

Fig. 4. AFM image of surface relief (a) and reflectance, transmittance and absorbance spectra for $43 \mathrm{~nm}$ gold film on the glass substrate $(b)$.

\subsection{SEIRA experiments on SPEV cells}

SEIRA spectra of SPEV cells deposited on the gold substrate from suspension and after single or double washing with aqueous solution are presented in Fig. 5a. In the process of cells deposition from the suspension on the gold substrate, the cells did not cover the substrate as a monolayer, but usually they form a thick layer. The SEIRA spectrum from the thick layer (Fig. 5a, curve 1) indicates the low intensity and pure band resolution due to excessive IR absorption for separated bands. The higher quality spectra of the cells were registered after washing (Fig. 5a, curve 2). After washing, the cells are arranged separately in a monolayer manner, at some distance from each other, which was confirmed by confocal microscopy (see Figs. 6, 7 and 8). Note, that with SEIRA spectroscopy we have registered signal from about 100 cells.

From Fig. 5b one can see differences in the spectra of the cells cultivated directly on the gold surface and

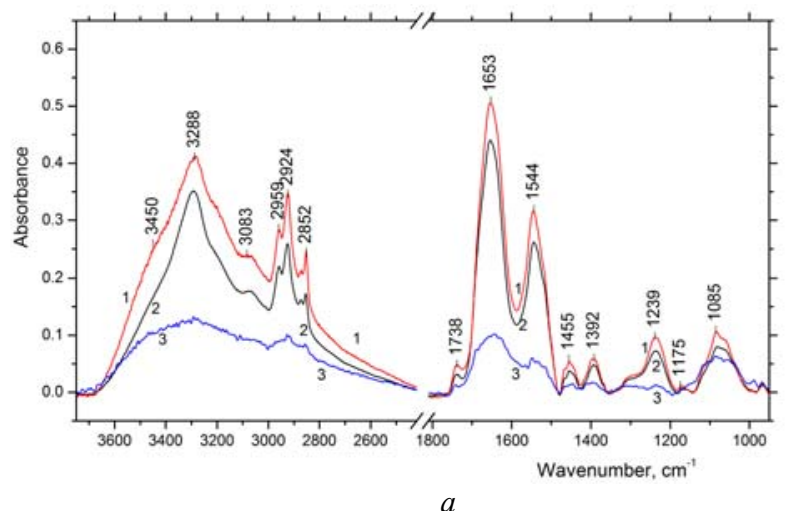

transferred to the gold substrate from suspension. The main spectral changes in SEIRA spectra of these samples were observed in the regions of $\mathrm{NH}, \mathrm{OH}, \mathrm{CH}_{2}-$ $\mathrm{CH}_{3}$ stretching vibration bands, Amid I and Amid II bands, as well as in the $900 \ldots 1300 \mathrm{~cm}^{-1}$ region. For the SPEV cells cultivated on the gold substrate, we have registered a shift of different bands by $4 \ldots 7 \mathrm{~cm}^{-1}$ to low frequencies, except for phosphate asymmetrical stretching band. Specifically, $\mathrm{CH}$ stretching vibration bands were shifted by $4 \mathrm{~cm}^{-1}$, Amid I - by $6 \mathrm{~cm}^{-1}$, Amid II - by $4 \mathrm{~cm}^{-1}$. In addition, in the spectra of SPEV cells cultivated on the gold substrate the relative intensity of Amid I and Amid II bands increases comparing with those of SPEV cells deposited on gold substrate. However, no change in the intensity or frequency of Amid A band near $3292 \mathrm{~cm}^{-1}$ was observed. The observed shifts of the bands position in the spectra of SPEV cultivated on the gold substrate in comparison with those of the cells grown on the glass substrate could be explained by more strong interaction

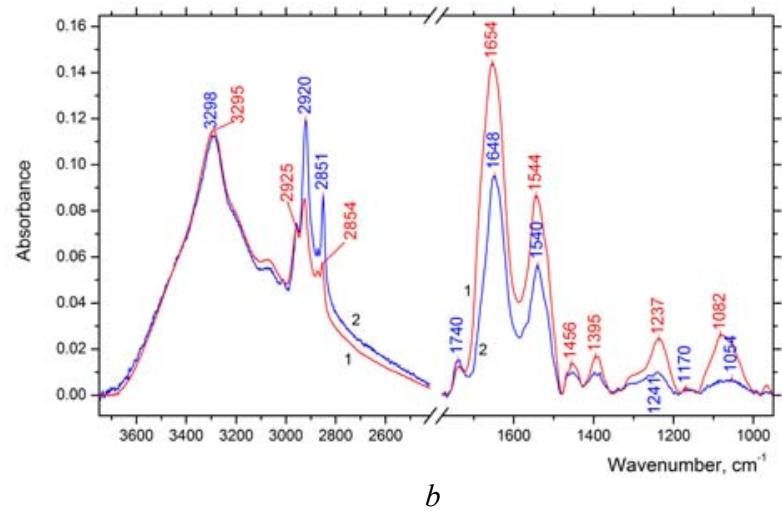

Fig. 5. a) SEIRA spectra of SPEV cells adsorbed from suspension on gold substrate just after deposition (3) and after single (2) or double (1) washing. b) SEIRA spectra of SPEV cells transferred to the gold substrate from suspension (1) and cultivated directly on the gold surface (2). 
between the cells and the gold substrate. Note that in any case the shifts of the IR bands position of the cellular components, DNA and proteins deposited on the gold substrate in comparison with those on the neutral substrate as $\mathrm{CaF}_{2}$ in our earlier papers $[1-4,8,9]$ are in accordance with the present results.

For the SPEV cells deposited from suspension, the conformation analysis of protein bands showed the preferable presence of $\alpha$-helix $\left(1653 \ldots 1654 \mathrm{~cm}^{-1}\right)$, and that for asymmetrical phosphates showed DNA presence in A-form $\left(1237 \ldots 1241 \mathrm{~cm}^{-1}\right)$. Thus, we have got an enhancement of IR absorption of SPEV cells deposited on the rough gold substrate (by about $15 \ldots 30$ times) for different vibrations and obtained high-quality spectra with good resolution.

\subsection{Confocal microscopy}

Fig. 6a demonstrates fluorescent images of the initial rough gold substrate at all excitation wavelengths used, namely, with the blue $(405 \mathrm{~nm})$, green $(488 \mathrm{~nm})$ and red $(633 \mathrm{~nm})$ laser lines. Observed fluorescence is in agreement with previous data of Boyd et al.[26], where the authors reported a fluorescence of a gold film with rough surface with characteristic peaks near $2.0 \mathrm{eV}$ $(0.62 \mu \mathrm{m})$ and $2.8 \mathrm{eV}(0.44 \mu \mathrm{m})$ excited by light source with $3.50 \mathrm{eV}(0.35 \mu \mathrm{m})$ or $4.67 \mathrm{eV}(0.27 \mu \mathrm{m})$. They arise from intra- and inter-band transitions of gold as well connected with recombination of holes and electrons [26]. This fluorescence can be enhanced by local electromagnetic field of plasmons excited at the gold rough surface $[27,28]$. Thus, gold substrate is a source of both proper fluorescence and enhanced local electromagnetic field that can effect on optical process near metal surface $[19,20]$. Fig. $6 \mathrm{~b}$ demonstrates that a gold-free area of glass substrate (black area down) and scratches on the gold surface do not show any fluorescence at excitation conditions specified. Clear glass looks like black spot under laser excitation in the confocal microscope contrary to the gold substrate that looks like bright spot and gives pronounced fluorescence.

Fig. 7 shows a good-quality confocal microscopy luminescent image of the SPEV cells cultivated on gold substrate excited using UV lamp $(365 \mathrm{~nm})$ and registered via FSet 01 filter. Different sub-cellular structures are clearly seen.

The SPEV and fibroblast cells cultivated or deposited on the gold substrate have a weak proper fluorescence under UV-lamp excitation (Fig. 8), which is a traditional image for confocal microscopy. Nevertheless, under excitation with UV radiation, a membrane and intracellular structures visualization can be achieved, when gold-covered substrate is used. The fluorescence from membrane structures is shifted to the long-wave region in comparison with intracellular components, and it simplifies the procedure of visualization. So, if the cells have no proper luminescence, one could register an image of the cells as a shadow from the cells due to fluorescence from the gold.

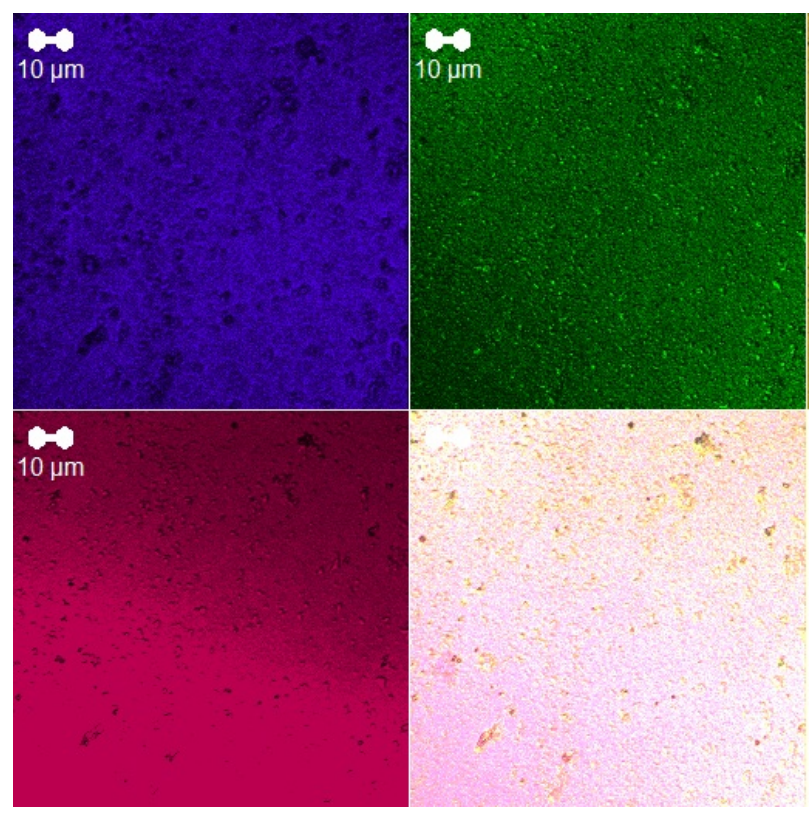

$a$

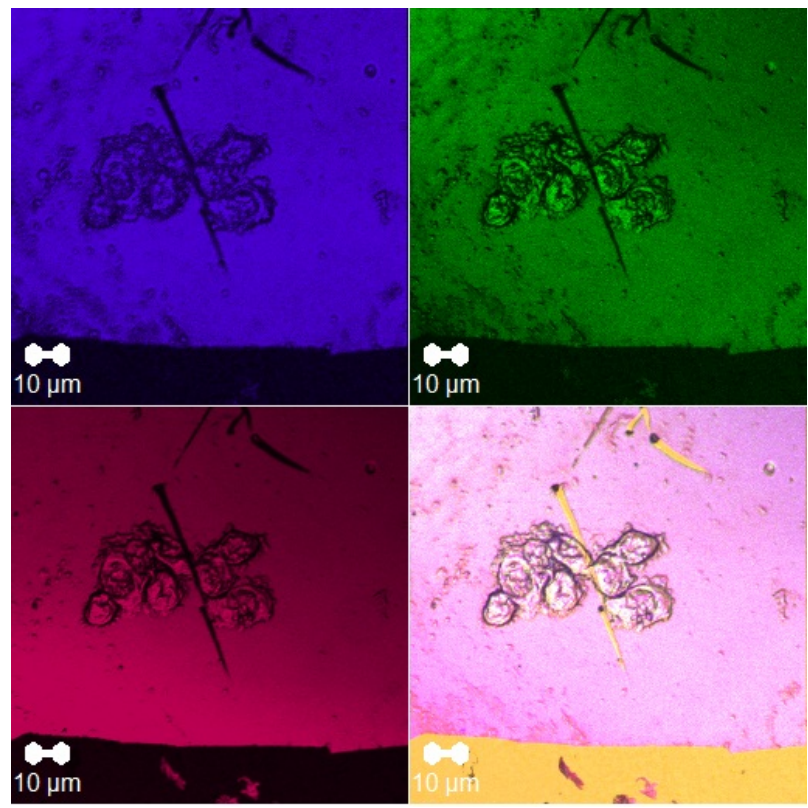

$b$

Fig. 6. Confocal microscopy fluorescent images of rough initial gold substrate excited with 405, 488 and $633 \mathrm{~nm}$ laser lines, registered with BP 420-480, BP 505-530 and LP $650 \mathrm{~nm}$ filters, respectively, presented by the dark blue, green and pink colors and their aggregated image marked with rose (a). Images of colony of the suspended cells on the gold rough substrate; the glass substrate and scratch on the gold look like black spots (b). Proper long pass filters were used for registration, magnification $63 \times$. 


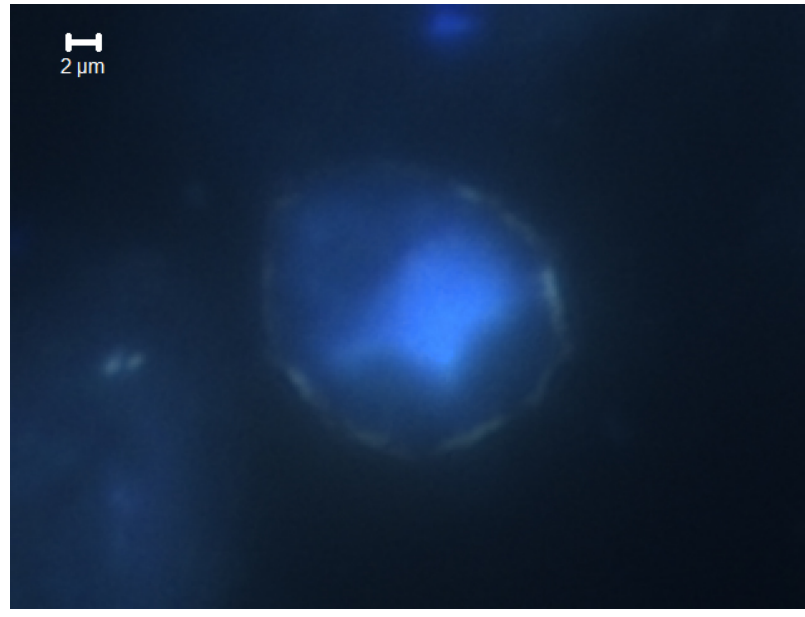

Fig. 7. Confocal microscopy luminescent image of the SPEV cells cultivated on gold substrate excited using UV lamp $(365 \mathrm{~nm})$ and registered via FSet 01 filter $($ Exc $=$ BP 365/12, emiss $=$ LP 397).

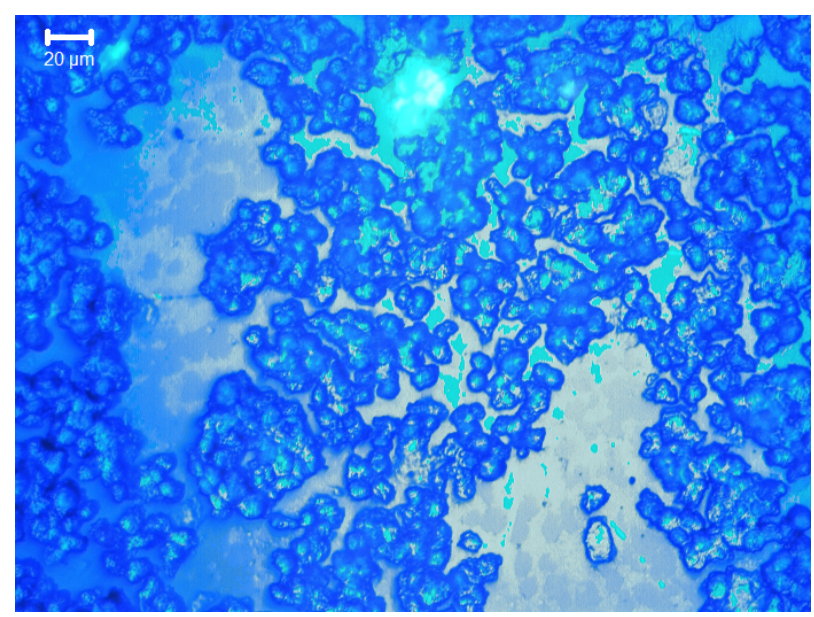

Fig. 8. Confocal microscopy fluorescent image of fibroblasts excited by UV lamp with FSet 01 filter $($ Exc $=$ BP 365/12, beamspl $=$ FT 395, emiss $=$ LP 397).

However, the excitation with laser provides better images of the cells in vivo on the gold-covered substrate (Fig. 9), which is not possible to get in the luminescent mode on glass coverslip without staining the cells. Under excitation with green or blue laser, the cells on the gold reveal better emission, and the obtained images are of the best quality, while the red laser excitation gives a picture of cells as a shadow. In the latter case, sometimes we can see a fragment of the interior fluorescence from the cells. In the conventional mode, PL from the stained cells is registered via band pass filters of BP 420-480, BP 505-530 and LP 650, and in this case PL is weaker than that with application of long pass filters. Quality of our cell images depends on PL of the gold surface stronger than on the type of filters.

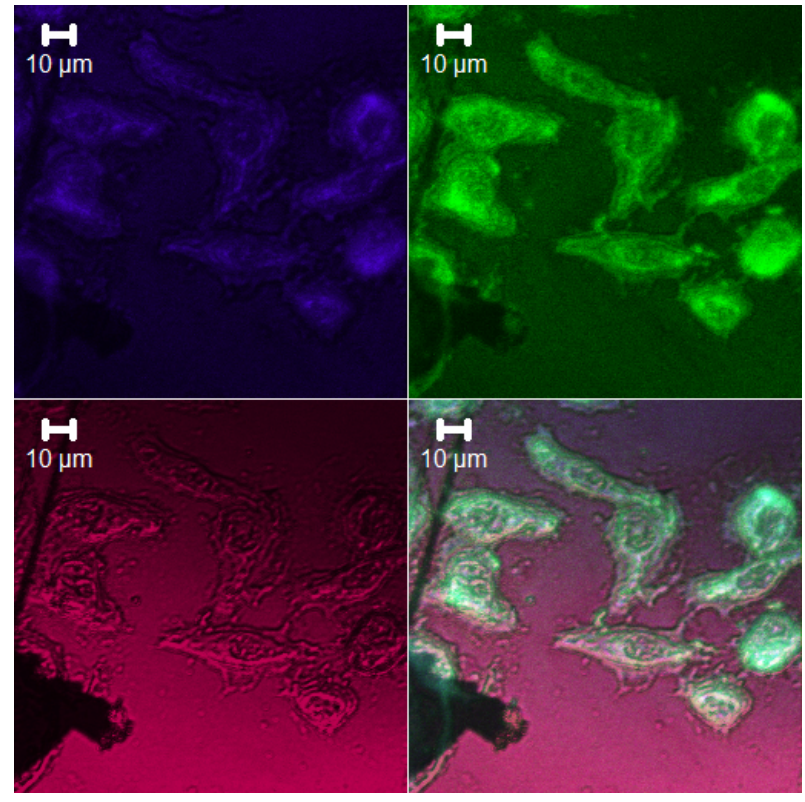

Fig. 9. Confocal luminescence images of SPEV cells on the gold substrate excited by 405, 488 and $633 \mathrm{~nm}$ laser lines and registered via long pass filters LP 420, LP 505, LP 650. Aggregated image is presented as well.

Time dependence of PL inherent to the SPEV cells at continuous excitation with UV lamp via the blue filter set FSet 01 in time series mode is presented in Fig. 10. The decrease in the intensity of PL by 2-3 times from cell for 60-70 s was observed. A gold substrate and a cell have different complex dependence of fluorescence kinetics. Kinetics of the PL decay requires a special study.

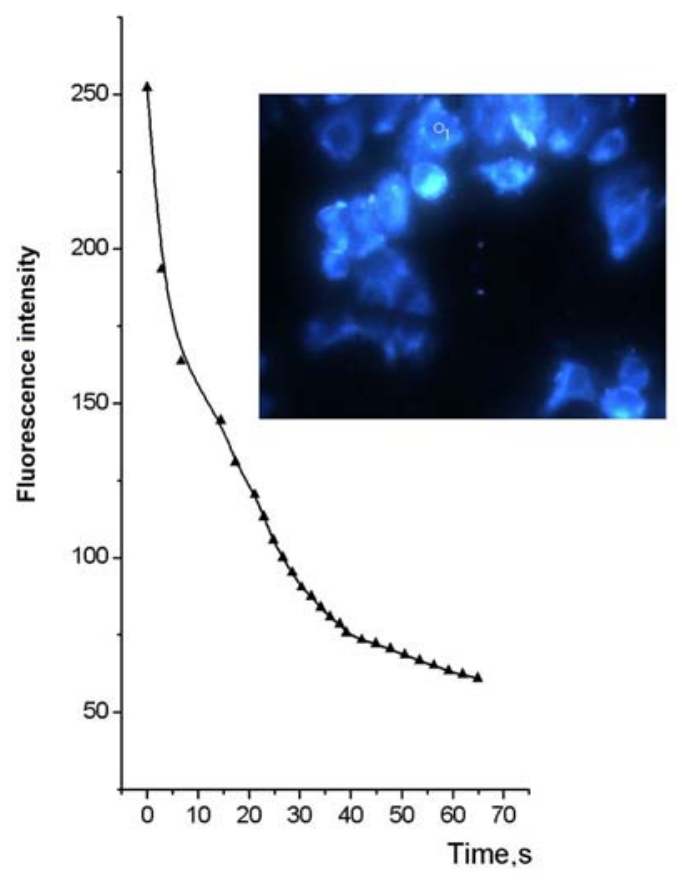

Fig. 10. Confocal microscopy image of the SPEV cells on the gold substrate and time dependence of fluorescence intensity from the cell (marked by circle 1). 
The origin of the above mentioned effects should be studied in details. Particularly, we could not propose preferable mechanism responsible for the fluorescence of cells and energy transfers. However, we can conclude that: 1) the rough semitransparent gold film substrate can enhance the proper fluorescence of the cells; 2) a potential of the confocal microscopy gives an opportunity to register a fluorescence from the biological cells in vivo on the gold enhancing substrate that is difficult to reach without labelling; 3) SEIRA spectroscopy of a cells monolayer on the rough gold substrate gives a possibility to analyze structural components of the cells with better resolution than conventional FTIR spectroscopy.

\section{Conclusions}

In this paper, a new approach for optical imaging, structural study and cell cultivation is proposed. Rough thin gold film on the glass coverslip was used as a substrate for SPEV cells growth and optical investigations. Such substrate evokes the enhancement of optical signals from biomolecules and biological cells located near nanostructured surface.

The enhancement factor of $15 \ldots 30$ was obtained for different molecular vibrations in the SEIRA spectra from monolayer of the SPEV cells cultivated on the rough gold as well as a low-frequency shift of about $2 \ldots .7 \mathrm{~cm}^{-1}$ for the majority of characteristic IR vibrations, as compared with that of cells adsorbed from suspension on the same substrate. For the first time, SEIRA spectra of monolayer of the SPEV cells deposited on the rough gold substrate were registered with better resolution than that obtained on monolayer of protein or other biopolymers.

The confocal microscopy contrast images of the SPEV cells on the rough gold substrate were obtained in laser fluorescence mode without cells labelling. The fluorescence of the rough gold surfaces and effects responsible for our findings have been discussed.

This approach opens new possibilities for visualization of the cells in vivo without staining.

Acknowledgements. The work was supported by Program of NAS of Ukraine No. VC 156 (2013-2016) and Institute for Problem of Cryobiology and Cryomedicine of the NASU (Volkova N.A.).

\section{References}

1. Dovbeshko G.I., Chegel V.I., Gridina N.Ya., Gnatyuk O.P., Shirshov Y.M., Tryndiak V.P., Todor I.M. Surface enhanced infrared absorption of nucleic acids on gold substrate. Semiconductor Physics, Quantum Electronics and Optoelectronics. 2001. 4, No. 3. P. 202-206.

2. Dovbeshko G.I., Chegel V.I., Gridina N.Ya., Gnatyuk O.P., Shirshov Y.M., Tryndiak V.P., Todor
I.M., Solyanik G.I. Surface enhanced infrared absorption of nucleic acids from tumour cells: an FTIR reflectance study. Biospectroscopy, USA. 2002. 67. P. 470-486.

3. Dovbeshko G.I. Molecular mechanism of interaction of biological molecule with nanostructures, ligands and low doses of ionizing and microwave irradiation. - Manuscript. The thesis applied for scientific degree of doctor of sciences in physics and mathematics on speciality 03.00.02Biophysics. - V.N. Karazin Kharkiv National University, Kharkiv, 2009.

4. Dovbeshko G.I., Gnatyuk O.P., Tryndiak V.P., Todor I.M., Solyanik G.I. Chehun V.F. in: Frontiers of Multifunctional Nanosystems. Eds. E. Buzaneva, P. Scharff. Amsterdam: Kluwer Academic Publ., 2002. P. 265-280.

5. Kuhne Ch., Steiner G., Fischer W.B., Salzer R. Surface enhanced FTIR spectroscopy on membrane. Fresenius J. Anal. Chem. 1998. 360. P. 750-754.

6. Ataka K. and Heberle J. Biochemical applications of surface enhanced infrared absorption spectroscopy. Anal. Bioanal. Chem. 2007. 388. P. 47-54.

7. Ataka K., Heberle J. Use of surface enhanced infrared absorption spectroscopy (SEIRA) to probe the functionality of a protein monolayer. Biopolymers. 2006. 82, No. 4. P. 415-419.

8. Dovbeshko G.I., Gnatyuk O.P., Chegel V.I., Shirshov Yu.M., Kosenkov D.V., Andreev E.A., Tajmir-Riahi H.A., Litvin P.M. Gold and colloidal gold surface influence on DNA conformational changes. Semiconductor Physics, Quantum Electronics and Optoelectronics. 2004. 7, No. 3. P. 318-325.

9. Dovbeshko G., Fesenko O., Gnatyuk O., Shtogun Ya., Woods L., Bertarione S., Damin A., Scarano D., Zecchina A. in: Carbon Nanotubes. Eds. J.M. Marulanda, In-Tech., 2010. P. 697-719.

10. Freshney R. Ian. Culture of Animal Cells: A Manual of Basic Technique and Specialized Applications. John Wiley \& Sons, 2015.

11. Birger M.O. (Ed.) The Handbook on Microbiological and Virologic Methods of Research. M.: Medicine, 1982.

12. Paddock S.W. (Ed.) Confocal Microscopy Methods and Protocols. Springer, 1999.

13. Hartstain A., Kirtley J.R., Tsang J.C. Enhancement of the infrared absorption of the molecular monolayers with thin metal overlayers. Phys. Rev. Lett. 1980. 45. P. 201-209.

14. Osawa M., Ikeda M. Surface-enhanced infrared absorption of p-nitro benzoic acid deposited on silver island films: contributions of electromagnetic and chemical mechanisms. J. Phys. Chem. 1991. 95. P. 9914-9919.

15. Kosobukin V.A. Collective modes in enhancement of external electric field on the surface of the metals. Doklady Akademii Nauk SSSR, Ser. Fiz. 1985. 49, No. 6. P. 1111-1120 (in Russian). 
16. Kosobukin V.A. Effect of enhancement of external electric field near metal surface and its manifestation in spectroscopy. Poverkhnost'. Fizika, khimiya, mekhanika. 1983. 12. P. 5-20 (in Russian).

17. Osawa M. in: Vol. 1. Handbook of Vibrational Spectroscopy. Eds. J.M. Chalmers, P.R. Griffiths. Chichester: Wiley, 2002. P. 785-800.

18. Suetaka W. Surface Iinfrared and Raman Spectroscopy: Methods and Applications. New York: Plenum Press, 1995.

19. Nishikawa Y., Fujiwara K., Ataka K., Osawa M. Surface-enhanced infrared external reflection spectroscopy at low reflective surfaces and its application to surface analysis of semiconductors, glasses, and polimers. Anal. Chem. 1993. 65. P. 556-562.

20. Aroca R.F., Ross D.J., Domingo C. Surfaceenhanced infrared spectroscopy. Appl. Spectrosc. 2004. 58. P. 324A-338A.

21. Lakowicz J.R., Shen B., Gryczynski Z., D’Auria I., Gryczynski S. Intrinsic fluorescence from DNA can be enhanced by metallic particles. Biochem. and Biophys. Res. Communs. 2001. 286. P. 875-879.
22. Lakowicz J.R. Radiative decay engineering: Biophysical and biomedical applications. Anal. Biochem. 2001. 298. P. 1-24.

23. Bondar V.V., Kuryk M.V. Exciton luminescence on the interface antracene-gold. J. Exp. Theor. Phys. 1980. 78, No. 1. P. 94-99.

24. Aspnes D.E., Kinsbron E., Bacon D.D. Optical properties of Au: Sample effects. Phys. Rev. B. 1980. 21. P. 3290-3299.

25. Maier S.A. Plasmonics: Fundamentals and Applications. Springer Science and Business Media LLC, 2007.

26. Boyd G.T., Yu Z.H., Shen Y.R. Photoinduced luminescence from the noble metals and its enhancement on roughened surfaces. Phys. Rev. B. 1986. 33, No. 6. P. 7923-7926.

27. Lumdee C., Yun B., Kik P.G. Effect of surface roughness on substrate-tuned gold nanoparticle plasmon resonances. Nanoscale. 2015. 7, No. 9. P. $1-6$.

28. Andersen S.K.H., Pors A., Bozhevolnyi S.I. Gold photoluminescence wavelength and polarization engineering. ACS Photonics. 2015. 2, No. 3. P. 432438. 